\title{
The role of artificial nutrition in gynecological cancer therapy
}

\author{
Magdalena Szewczuk ${ }^{1}$ Emilia Gasiorowska² ${ }^{2}$ Konrad Matysiak³ ${ }^{3}$ Ewa Nowak-Markwitz² \\ ${ }^{1}$ Department of Gastroenterology, Clinical Hospital H. Swiecicki University of Medical Sciences, Poznan, Poland \\ ${ }^{2}$ Department of Gynecology, Obstetrics and Gynecologic Oncology, Division of Gynecologic Oncology, \\ Poznan University of Medical Sciences, Poznan, Poland \\ ${ }^{3}$ Centre for Intestinal Failure, Department of General, Endocrinology and Gastroenterological Surgery, \\ Poznan University of Medical Science, Poznan, Poland
}

\begin{abstract}
Cancer patients are at risk of developing malnutrition from underlying disease as well as from cancer treatment. Moreover, weight loss is considered as a predictive factor for disease progression and shorter survival time. As many as $10-20 \%$ of patients with cancer die from the results of malnutrition, instead of from the cancer itself. In the case of cancer-related malnutrition, it is necessary to quickly implement individualized nutritional support depending on the type and stage of the disease, metabolic changes, the patient's condition, expected survival and the function of the gastrointestinal tract. Artificial nutrition reduces the side effects of chemotherapy and improves immunity. Perioperatively it reduces the risk of infection, facilitates wound healing and shortens the length of hospitalization, thereby reducing the costs of the treatment. Initially, a malnourished patient, without gastrointestinal dysfunction, qualifies for nutritional counseling. When the energy needs cannot be met by normal feeding, nutritional supplements, taken orally, are recommended. The next step is to feed the patient by nasogastric tube or percutaneous endoscopic gastrostomy. Parenteral nutrition, which results in more side effects, is only started when enteral nutrition is insufficient to ensure adequate nutritional status or in cases of gastrointestinal tract obstruction. The benefit of parenteral nutrition is that it especially provides for those patients with gynaecological cancer who have radiation-induced intestinal damage and post-surgical complications such as short bowel syndrome. Palliative nutrition must to relieve hunger and thirst. Nutritional interventions should be individualized and focused on the changing nutrient needs of the patient and should be supported by physical activity. Regular assessment of the nutritional status of the patient should be an inherent element of the oncological treatment.
\end{abstract}

Key words: parenteral nutrition; gynecological cancers; malnutrition; enteral nutrition; nutritional treatment

Ginekologia Polska 2019; 90, 3: 167-172

\section{INTRODUCTION}

Cancer patients are at risk of developing malnutrition from the underlying disease as well as from the cancer treatment. In addition, weight loss is considered as a predictive factor for disease progression and indicates shorter survival times. It is known that $10-20 \%$ of patients with cancer die from the results of malnutrition, instead of from the cancer itself [1]. In 2012, studies showed that doctors underestimate how malnutrition influences patients' quality of life and the cancer treatment itself [1]. The data shows that 20-70\% of oncological patients are malnourished and this applies more often to older patients; but only $30-60 \%$ of patients with a high risk of developing malnutrition are treated [2].
This is caused by a lack of knowledge of the guidelines for nutritional treatment in cancer which are an important part of oncological treatment. In Poland, female patients suffering from gynecological cancers comprise $11 \%$ of all patients treated in the Nutritional Centers of Poznan alone. These are mainly patients in the terminal stage of the disease, following several methods of treatment, and when the disease is advanced and life expectancy is not long. Only one fourth of them is treated, especially with parenteral nutrition, for longer than four months. It is necessary to make screening tests among all patients with reproductive organ cancer, in order to estimate the risk of them developing malnutrition. This should be done every time 
a patient is qualified for treatment, and tests should include BMI, estimated weight loss, and muscle mass. Blood tests should be also considered. The European Society for Clinical Nutrition and Metabolism (ESPEN) analysed all the causes and effects of malnutrition in cancer patients and have provided specific guidelines for such cases [3]. Nutritional interventions should be individualized and focused on the changing nutrient needs of the patient and on the reduction of inflammation marker levels and should be supported by physical activity [4].

\section{CURRENT STATE OF KNOWLEDGE}

\section{Mechanism of malnutrition}

Among cancer patients the most frequent causes of malnutrition are: loss of appetite, food intolerance, nausea, vomiting, symptoms associated with local tumour growth, hypoalbuminemia, anaemia, as well as side effects of the oncological treatment [5]. Chemotherapy may cause gastrointestinal mucositis, mouth ulcerations, secondary haemorrhage, diarrhoea, dysgeusia (taste disturbance), and nausea. All the above lead to a reduction of food intake and increased risk of malnutrition [6]. Similarly, the adverse effects of radiotherapy mainly affect surrounding tissues, and in patients receiving treatment for cervical cancer or endometrial cancer these adverse effects are enteritis and malabsorption disorder [3,5]. The tumour itself, causes an increased inflammatory response, mediated by IL-6, which plays an important role in the development of malnutrition, with catabolic effects [7]. Pro-inflammatory cytokines cause malabsorption of nutrients and interfere with the metabolism of carbohydrates, fats and proteins, and they affect the central appetite control system leading to anorexia. Cytokines also affect hepatic overproduction of pro-inflammatory acute phase proteins, which leads to the worsening metabolism of anticancer drugs and increases their toxicity. Activation of inflammatory factors causes the breakdown of tissues and, as a result, weight loss and the reduction of muscle mass [7]. Loss of muscle mass is associated with worsened prognosis and also occurs in obese people. Among those patients, despite having a high BMI, muscle mass loss also occurs, along with all the malnutrition consequences $[5,8]$. $\mathrm{BMI}$ is a less valuable indicator, because obesity is becoming a more serious problem. Doctors need to pay more attention to recent weight loss or weight loss within a short period, and to the patient's reduced food intake.

\section{Diagnosis of malnutrition}

Weight loss is an important sign of malnutrition [8]. Special scales are used to assess the patient's nutritional status. In hospitals, the most common ways to assess nutritional status are the nutritional risk score (NRS) (Tab. 1) and the subjective global assessment (SGA) of nutritional status [9]. Further advanced assessments of nutritional status are performed by body composition tests using bioelectrical impedance analysis, computer tomography, magnetic resonance imaging, biochemical tests and anthropometrical indices, such as measuring the thickness of the skin fold over the triceps muscle or the circumference of arm muscles [9]. According to ESPEN, malnutrition is diagnosed when the patient's BMl is $<18.5 \mathrm{~kg} / \mathrm{m}^{2}$ or when the patient reports

Table 1. Screening of risk assessment related to malnutrition - NRS 2002 adults (above 18 years of age)

\begin{tabular}{|c|c|c|c|}
\hline \multicolumn{2}{|c|}{ Impaired nutritional status } & \multicolumn{2}{|c|}{$\begin{array}{l}\text { Severity of the disease } \\
\text { ( increase in requirements ) }\end{array}$} \\
\hline Score $=0$ & normal nutritional status & Score $=0$ & normal nutritional requirement \\
\hline Mild Score $=1$ & $\begin{array}{l}\text { weight loss }>5 \% \text { in } 3 \text { months or food intake } \\
\text { below } 50-75 \% \text { of normal requirement in } \\
\text { preceding week }\end{array}$ & Mild Score = 1 & $\begin{array}{l}\text { e.g. hip fracture, chronic diseases, especially in } \\
\text { patients with acute complications (eg cirrhosis } \\
\text { of the liver, COPD), radiotherapy }\end{array}$ \\
\hline Moderate Score = 2 & $\begin{array}{l}\text { weight loss }>5 \% \text { in } 2 \text { months or BMI } 18.5- \\
20.5+\text { impaired general condition or food } \\
\text { intake } 25-50 \% \text { of normal requirement in } \\
\text { preceding week }\end{array}$ & Moderate Score = 2 & $\begin{array}{l}\text { major abdominal surgery, stroke, elderly } \\
\text { patients - long-term treatment, postoperative } \\
\text { renal failure, chemotherapy }\end{array}$ \\
\hline Severe Score $=3$ & $\begin{array}{l}\text { weight loss }>5 \% \text { in } 1 \text { month or } \\
\mathrm{BMI}<18.5+\text { impaired general condition or } \\
\text { food intake }=0-25 \% \text { of normal requirement in } \\
\text { preceding week. }\end{array}$ & Severe Score = 3 & $\begin{array}{l}\text { head injury, bone marrow transplantation, } \\
\text { intensive care patients }\end{array}$ \\
\hline \multicolumn{4}{|c|}{ if $>70$ years: add 1 to total score above } \\
\hline \multicolumn{2}{|l|}{ Score: } & Score: & Total points: \\
\hline
\end{tabular}

Instructions:

1. select one appropriate degree of disturbance of the state of nutrition and the severity of the disease

2. sum points

Score:

$\geq 3$ - indicated nutritional treatment

$<3-$ consider a conservative procedure, repeat the test in a week 
unintentional weight loss ( $>10 \%$ over an unknown period or $>5 \%$ over 3 months), and in connection with the patient's $\mathrm{BMI}(<20$ in patients under 70 years and $<22$ in patients over 70 years), or with a low fat-free mass index (FFMI) of $<15 \mathrm{~kg} / \mathrm{m}^{2}$ for women and $<17 \mathrm{~kg} / \mathrm{m}^{2}$ for men. (Fig. 1). We also assess CRP and albumin levels using the Glasgow Prognostic Score. We identify the nutrition as inadequate when the patient has not eaten for a week or when the food intake covers less than $60 \%$ of the energy demands over 1-2 weeks. A change in appetite is the first symptom of being at risk of malnutrition, and normal nutrition does not meet the body's needs in cancer. Nutrition support must be introduced gradually to avoid refeeding syndrome, and especially for patients with large deficits, because it can lead to electrolyte, hormonal and metabolic changes and consequently can cause neurological disorders and cardiological complications [10] (Fig. 1).

\section{Consequences of malnutrition}

The consequences of malnutrition in oncological patients are weight loss and muscle mass loss, weakening of the immune system, increased frequency of infections, more complications and less tolerance to chemotherapy. Malnutrition is associated with a greater number of complications of chemotherapy and radiotherapy, which may even lead to the necessity to stop treatment and reducing its effectiveness. It has been clearly shown that malnutrition influences the faster progression of the disease, higher mortality rates and poorer quality of life. The consequences of malnutrition are also pain, weakness and depression. Patients with stable body weight have a longer survival time [11]. Nutritional status is an important predictor of the treatment's tolerance and of increased mortality. Therefore, it is important not only to recognize malnutrition, but also to identify and monitor patients at risk of its occurrence, in order to start early nutritional treatment [12-14].

\section{Artificial nutrition}

The goal of nutritional treatment in cancer patients is to prevent any further deterioration of their nutritional status, as well as to support the oncological treatment [15]. It is a supportive treatment that improves the patient's functional ability and overall fitness state, increases the body's immune system, improves tolerance to chemotherapy and reduces its side effects. However, in order to achieve the desired goals, it must be introduced early enough, and if this is achieved, it reduces the risk of infections, enables wound healing, shortens hospitalization and reduces the costs of treatment. Physical activity is also important and prevents loss of muscle mass. Artificial nutrition includes nutrient supplements introduced via the gastrointestinal tract (oral or enteral) or by intravenous line. (Fig. 1). If an oncological patient with an expected survival time longer than several months who does not have gastrointestinal dysfunctions, is unable to cover their energy demands with a normal diet, nutritional treatment is induced. This treatment is based on the diagnosis of the type and stage of the disease, its severity, the type of treatment planned, as well as taking into consideration the individual preferences and general condition of the patient. The first step is dietary counselling. If this is insufficient, the next step is the introduction of oral nutrition supplements followed by feeding the patient by nasogastric tube or percutaneous endoscopic gastrostomy [16]. Parenteral nutrition, which has more side effects than the abovementioned treatments, is only started when enteral nutrition is insufficient to ensure the patient's adequate nutritional status and when malnutrition will shorten the survival time compared with the prognosis of death due to cancer disease itself. (Fig. 1).

\section{Parenteral nutrition in gynecologic oncology}

Around $50 \%$ of patients referred from gynaecological oncology units for parenteral nutrition treatment are patients suffering from cervical or endometrial cancer with radiation-induced enteritis and secondary malabsorption syndrome. Acute radiation-induced enteropathy occurs within a few days after the therapy and affects only the mucous membrane. The subacute condition occurs during the first year after radiotherapy and the mucous and submucous membrane are affected. However, the chronic condition occurs several years after radiotherapy and affects all layers of the intestine wall. The risk of enteropathy increases with age, co-morbidities, and after previous surgical procedures. About $80 \%$ of patients treated with radiotherapy focused on their pelvic area experience side effects associated with their digestive system and suffer weight loss. About $20 \%$ of them will develop chronic intestinal inflammation after radiotherapy. Intestinal failure develops in about 5\% and this is a group that should be treated with parenteral nutrition as they will benefit the most from this type of nutrition. Nutritional support, preferably enteral nutrition, during the entire treatment of patients with cervical cancer and endometrial cancer, will either avoid or reduce side effects and the interruptions of treatment that are caused by the side effects of radiotherapy [17].

The conditions for patients with advanced cancer qualifying for parenteral nutrition are: expected overall survival time $>3$ months, $>50$ points in the Karnofsky score and no irreversible damage of the liver, kidneys and lung functions. The main indications for parenteral nutrition in gynecologic oncology include:

- obstruction of the gastrointestinal tract in advanced, metastatic ovarian cancer (secondary dysphagia, when enteral nutrition is impossible); 


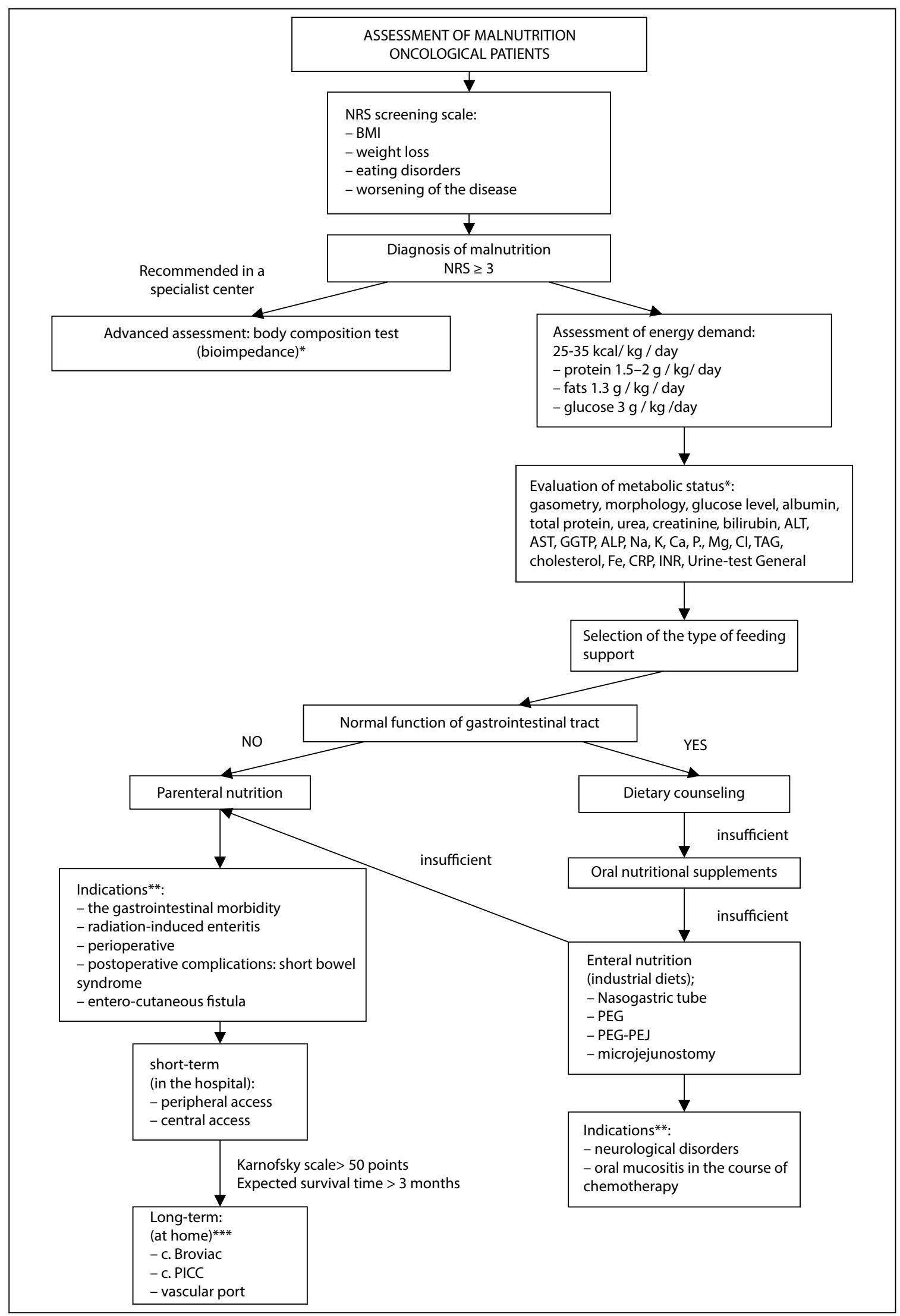

Figure 1. A brief description of the procedure in the event of suspected malnutrition. PEG-PEJ - percutaneous endoscopic gastrostomypercutaneous endoscopic jejunostomy; PEG - percutaneous endoscopic gastrostomy. * Marking 1-2 a week or more often depending on the needs and type of disorder. ${ }^{* *}$ The most common selected indications in oncological gynecology. ${ }^{* *}$ Referral to a Nutritional Treatment Center 
- malabsorption syndrome among patients with enteritis after radiotherapy (cervical and endometrial cancer);

- post-surgical complications (short-bowel syndrome, entero-cutaneous fistulas);

- perioperative nutrition support to reduce the number of complications.

Parenteral nutrition can be divided into 2 parts: short-term, used in the perioperative period in malnourished patients, which reduces perioperative risk and strengthens the immunological response; and long-term, used in the home environment with patients with chronic gastrointestinal insufficiency. Different types of central venous lines are used: Broviac catheter-central venous catheter with subcutaneous tunnelling, inserted through the jugular vein, the subclavian vein or the femoral vein; PICC catheter-peripherally inserted central catheter, inserted through the cephalic vein or through the basilic vein; and ports. However, in the case of a port, there is a higher risk of infections compared with the former two methods. All three methods mentioned above can also be used for administering chemotherapy while maintaining the principles of high sterility.

\section{Artificial nutrition in palliative care}

Often patients are referred for nutritional treatment too late, when they are disqualified from other forms of treatment and cachexia is irreversible. Cachexia is considered to be a condition that is resistant to treatment in the last stages of life. In palliative care, non-invasive feeding support, adequate for the patient's needs, is administered, with the goal of improving the patient's comfort and quality of life [18]. Palliative nutrition must relieve hunger and thirst. Parenteral nutrition has no proven efficacy in this group of patients. Only patients in an otherwise good general condition who have a gastrointestinal obstruction will gain some benefits from this method of nutrition. The psychological aspect is also very important. Patients with advanced gynaecological cancer complicated by intestinal obstruction live for 40-93 days [19]. These patients are often referred for parenteral nutrition, but this treatment usually only lasts from a few days to several weeks [19]. It should also be remembered that, according to ESPEN, parenteral nutrition is associated with a greater risk of side effects, which occur in $4-54 \%$ of patients, including: infections associated with the feeding port, deep vein thrombosis, deterioration of liver functions, worsening organ failure, and reduced quality and comfort of life through increasing amounts of effusion fluids in patients with ascites or pleural effusion.

\section{SUMMARY}

Cancer patients are at risk of developing malnutrition from the underlying disease as well as from their cancer treatment. Malnutrition increases the number of side ef- fects of chemotherapy and shortens patient survival time. The main goals of artificial nutrition, whether oral, enteral or parenteral, are to prevent further weight loss, improve muscle strength, restore lost tissues and subsequently cause weight gain. The benefit of parenteral nutrition is that it especially provides for patients who have radiation-induced intestinal damage and post-surgical short bowel syndrome. Palliative nutrition must relieve hunger and thirst. Regular assessment of the nutritional status of the patient should be an inherent element of the oncological treatment.

\section{Conflicts of Interest}

The authors declare that there are no conflicts of interest.

\section{Contributions of Authors}

We confirm that all the co-authors have been included, have contributed to the final manuscript and have approved it. MS and EG designed the study, analyzed the data, wrote the manuscript and prepared figures for this manuscript. ENM and KM critically reviewed the manuscript.

\section{REFERENCES}

1. Gyan E, Raynard B, Durand JP, et al. NutriCancer2012 Investigator Group NutriCancer2012 Investigator Group. Malnutrition in Patients With Cancer. JPEN J Parenter Enteral Nutr. 2017 [Epub ahead of print]; 42(1): 255260, doi: 10.1177/0148607116688881, indexed in Pubmed: 28135422.

2. Pressoir M, Desné S, Berchery D, et al. Prevalence, risk factors and clinical implications of malnutrition in French Comprehensive Cancer Centres. Br J Cancer. 2010; 102(6): 966-971, doi: 10.1038/sj.bjc.6605578, indexed in Pubmed: 20160725.

3. Arends J, Baracos V, Bertz H, et al. ESPEN expert group recommendations for action against cancer-related malnutrition. Clin Nutr. 2017; 36(5): 1187-1196, doi: 10.1016/j.clnu.2017.06.017, indexed in Pubmed: 28689670.

4. Lacau St Guily J, Bouvard É, Raynard B, et al. NutriCancer: A French observational multicentre cross-sectional study of malnutrition in elderly patients with cancer. J Geriatr Oncol. 2018; 9(1): 74-80, doi: 10.1016/j. jgo.2017.08.003, indexed in Pubmed: 28888553.

5. Arends J, Bachmann P, Baracos V, et al. ESPEN guidelines on nutrition in cancer patients. Clin Nutr. 2017; 36(1): 11-48, doi: 10.1016/j. clnu.2016.07.015, indexed in Pubmed: 27637832.

6. Wong PW, Enriquez A, Barrera R. Nutritional support in critically il patients with cancer. Crit Care Clin. 2001; 17(3): 743-767, indexed in Pubmed: 11525056.

7. Laird BJ, McMillan DC, Fayers $\mathrm{P}$, et al. The systemic inflammatory response and its relationship to pain and other symptoms in advanced cancer. Oncologist. 2013; 18(9): 1050-1055, doi: 10.1634/theoncologist.2013-0120, indexed in Pubmed: 23966223

8. Johns N, Stephens NA, Fearon $\mathrm{KCH}$. Muscle wasting in cancer. Int J Biochem Cell Biol. 2013; 45(10): 2215-2229, doi: 10.1016/j.biocel.2013.05.032, indexed in Pubmed: 23770121.

9. Kondrup J, Allison SP, Elia M, et al. Educational and Clinical Practice Committee, European Society of Parenteral and Enteral Nutrition (ESPEN). ESPEN guidelines for nutrition screening 2002. Clin Nutr. 2003; 22(4): 415-421, indexed in Pubmed: 12880610.

10. Aaldriks AbA, van der Geest LGM, Giltay EJ, et al. Frailty and malnutrition predictive of mortality risk in older patients with advanced colorectal cancer receiving chemotherapy. J Geriatr Oncol. 2013; 4(3): 218-226, doi: 10.1016/j.jgo.2013.04.001, indexed in Pubmed: 24070460.

11. Fearon K, Arends J, Baracos V. Understanding the mechanisms and treatment options in cancer cachexia. Nat Rev Clin Oncol. 2013; 10(2): 90-99, doi: 10.1038/nrclinonc.2012.209, indexed in Pubmed: 23207794.

12. Lach K, Peterson SJ. Nutrition Support for Critically III Patients With Cancer. Nutr Clin Pract. 2017; 32(5): 578-586, doi: 10.1177/0884533617712488, indexed in Pubmed: 28633000. 
13. Lembeck ME, Pameijer CR, Westcott AM. The Role of Intravenous Fluids and Enteral or Parenteral Nutrition in Patients with Life-limiting Illness. Med Clin North Am. 2016; 100(5): 1131-1141, doi: 10.1016/j. mcna.2016.04.019, indexed in Pubmed: 27542432.

14. Cotogni P. Enteral versus parenteral nutrition in cancer patients: evidences and controversies. Ann Palliat Med. 2016; 5(1): 42-49, doi: 10.3978/j. issn.2224-5820.2016.01.05, indexed in Pubmed: 26841814.

15. Chow R, Bruera E, Chiu L, et al. Enteral and parenteral nutrition in cancer patients: a systematic review and meta-analysis. Ann Palliat Med. 2016; 5(1): 30-41, doi: 10.3978/j.issn.2224-5820.2016.01.01, indexed in Pubmed: 26841813.

16. Orrevall Y. Parenteral nutrition in the elderly cancer patient. Nutrition. 2015; 31(4): 610-611, doi: 10.1016/j.nut.2014.11.006.
17. Henson CC, Burden S, Davidson SE, et al. Nutritional interventions for reducing gastrointestinal toxicity in adults undergoing radical pelvic radiotherapy. Cochrane Database Syst Rev. 2013(11): CD009896, doi: 10.1002/14651858.CD009896.pub2, indexed in Pubmed: 24282062.

18. Dev R, Dalal S, Bruera E. Is there a role for parenteral nutrition or hydration at the end of life? Curr Opin Support Palliat Care. 2012; 6(3): 365-370, doi: 10.1097/SPC.0b013e328356ab4a, indexed in Pubmed: 22801468.

19. Prevost V, Grach MC. Nutritional support and quality of life in cancer patients undergoing palliative care. Eur J Cancer Care (Engl). 2012 21(5): 581-590, doi: 10.1111/j.1365-2354.2012.01363.x, indexed in Pubmed: 22574646. 